\title{
Cardiac tamponade as the initial presentation of malignancy: is it as rare as previously supposed?
}

\author{
K.W. Muir' and J.C. Rodger
}

Monklands District General Hospital, Monkscourt Avenue, Airdrie ML6 OLJ and 'University Department of Medicine and Therapeutics, Gardiner Institute, Western Infirmary, Dumbarton Road, Glasgow G11 6NT, UK

Summary: Advanced malignant disease frequently involves the heart and pericardium, and pericardial effusion is a common postmortem finding in such patients. Identification of pericardial effusions in life is uncommon, however, even when symptomatic. Cardiac tamponade occurring as the first presentation of malignancy appears to be uncommon.

We present five cases of cardiac tamponade due to undiagnosed malignancy which presented to a general medical unit over 18 months. The availability of echocardiography was an important factor in correct diagnosis, since clinical features were non-specific. Bronchial adenocarcinoma was the cause in three of the five cases.

Review of the literature confirms adenocarcinomas of the bronchus as the most common cause of this complication. The majority of cases have presented with large volume, haemorrhagic effusions, and cytology (with or without carcinoembryonic antigen measurement) was diagnostic in most patients. Immediate treatment with subxiphoid pericardiotomy is recommended; the role of balloon catheter pericardiotomy remains to be established. Combined chemotherapy and radiotherapy appears to extend survival, which in some cases may be prolonged.

We recommend that early echocardiography should be obtained in all patients presenting with apparent cardiac failure, since early treatment of malignant effusions provides symptomatic relief.

\section{Introduction}

Pericardial effusions associated with metastases to the heart or pericardium are a frequent finding at postmortem in malignant disease. ${ }^{1}$ The presentation of malignancy with cardiac tamponade appears to be uncommon. A literature search using Medline CD ROM, followed by a review of the references in each paper identified, revealed 73 cases of malignancy presenting as cardiac tamponade that have been reported since 1935 . We wish to report five cases of cardiac tamponade presenting to a district general hospital over a period of 18 months in which tamponade was the first manifestation of malignant disease. We suggest that malignant cardiac tamponade may be a more common mode of presentation of malignancy than has been previously supposed, and review the literature.

\section{Case reports}

Cardiac tamponade was diagnosed on the basis of right ventricular diastolic collapse on echocardio-

Correspondence: K.W. Muir, M.R.C.P.

Accepted: 28 April 1994 graphy, which was present in all cases at first examination. Details of the cases are given in Table I. Dyspnoea had acutely worsened in all cases at time of admission but many patients had symptoms for some time prior to the acute event. An example history is outlined for illustration.

Case 2

A 50 year old man presented with acute worsening of dyspnoea after a 6 week history of anorexia, weight loss, exertional dyspnoea, night sweats, non-productive cough and vague left-sided chest discomfort. He had no significant past medical history and smoked 30 cigarettes daily. On examination he had a small left pleural effusion and tender hepatomegaly of five finger breadths below the costal margin.

Blood tests revealed hyponatraemia (serum sodium was $129 \mathrm{mmol} / \mathrm{l}$ ) and elevated transaminases. Retrospective electrocardiograph (ECG) review found electrical alternans. Chest X-ray showed a globular heart with small left pleural effusion. On echocardiography there was a large pericardial effusion with features of tamponade, and $950 \mathrm{ml}$ of haemorrhagic fluid was aspirated. 


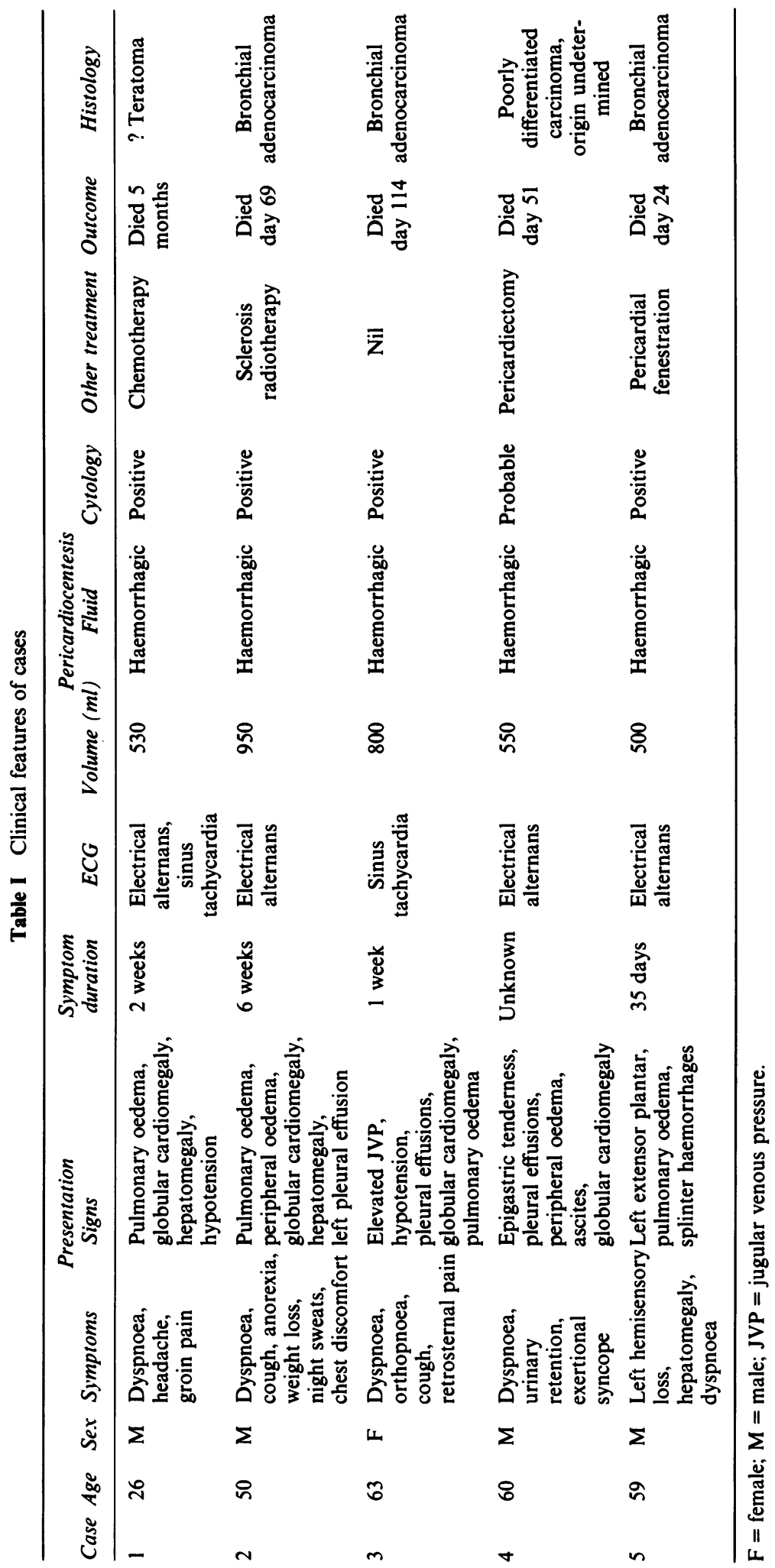


a

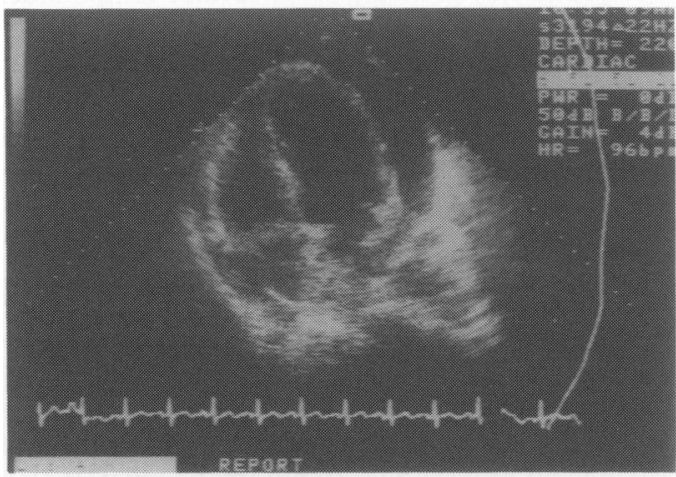

b

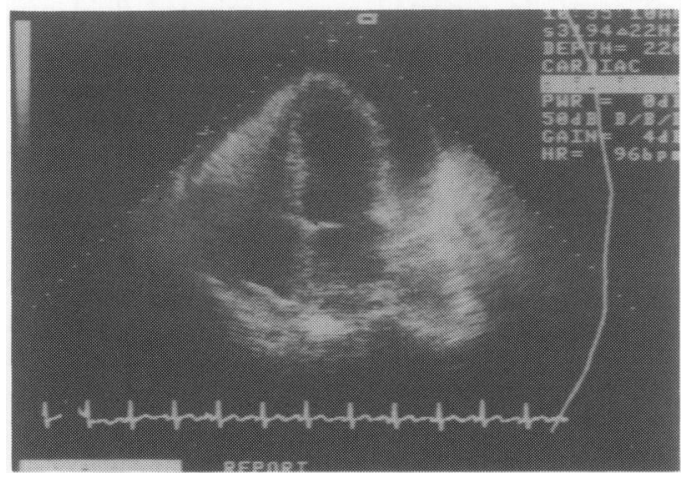

Figure 1 Two-dimensional trans-thoracic four chamber view echocardiograms in (a) systole and (b) diastole from case 5 , demonstrating right ventricular diastolic collapse.

Cytology demonstrated metastatic adenocarcinoma cells.

$\mathrm{He}$ required several further pericardiocenteses and was sent for radiotherapy following unsuccessful attempted sclerosis of the pericardial space. The effusion did not subsequently require to be tapped.

Prostatic acid phosphatase, carcinoembryonic antigen, alpha fetoprotein and beta human chorionic gonadotrophin levels were normal. A left hilar mass developed, and the primary tumour was assumed to be lung.

He was well enough to be discharged briefly from hospital but was readmitted with increasingly severe dyspnoea. There was no echocardiographic evidence of tamponade on this occasion. He deteriorated steadily and died 69 days after initial presentation.

\section{Discussion}

Metastatic spread of tumours to the heart or pericardium, causing pericardial effusion, is found in $11.6 \%$ of patients with known malignancy at postmortem examination. ${ }^{1}$ It is much less common to identify such effusions during life: in one study, only $30 \%$ of patients with symptoms referable to their pericardial disease were correctly identified antemortem. ${ }^{2}$ The tumour types most commonly found at postmortem to give rise to pericardial metastases were lung $(37 \%)$, breast $(22 \%)$, haematological malignancies $(17 \%)$ and melanoma (3\%). ${ }^{1}$ Cardiac tamponade is well recognized but rare in the course of advanced malignant disease. However, $16 \%$ of patients with autopsy-identified pericardial metastases had features of tamponade when reviewed restrospectively, despite a lack of clinical suspicion. ${ }^{2}$ Cardiac tamponade has previously been considered to be rare as a presenting feature of malignancy but epidemiological evidence regarding the true incidence of this mode of presentation is lacking. Fraser et al. reviewed the literature in 1980 and identified 26 cases, adding three of their own. ${ }^{3}$ Since that time, further case reports and small series have been added, ${ }^{4-11}$ bringing the total number of cases in the literature to 73. Most series are reported from oncology or cardiothoracic surgical units and we believe our report to contain the largest number of cases identified outside a specialist unit. It is undoubtedly the case that ready availability of echocardiography was the main factor in identifying these patients.

Tumour types and frequencies for all reported cases are detailed in Table II. The mean age of presentation was 50 years and there was a male predominance ( 55 males). Review of the literature shows that in $60 \%$ of cases, the primary site was lung. A large proportion of these were adenocarcinomas, with the more common sqamous carcinomas being comparatively rare as a cause of tamponade. Overall, adenocarcinomas of any origin accounted for $54 \%$ of all cases.

Table II Tumour types presenting as cardiac tamponade reported in literature, 1935-1992 $(n=78)$

Tumour type Number Percentage

Adenocarcinoma lung

Squamous carcinoma lung

Bronchoalveolar carcinoma

Other bronchial

Mesothelioma

Adenocarcinoma, unknown primary

Gastrointestinal tract

Leukaemia, lymphoma

Breast

Ovarian carcinoma

Other

$\begin{array}{rr}29 & 37 \\ 11 & 14 \\ 4 & 5 \\ 3 & 4 \\ 3 & 4 \\ & \\ 8 & 10 \\ 4 & 5 \\ 7 & 9 \\ 1 & 1 \\ 2 & 3 \\ 6 & 8\end{array}$


The development of tamponade is dependent on both the fluid volume and the rate of accumulation; the majority of malignant effusions presenting as tamponade are large, the average aspirate from one series being $1,200 \mathrm{ml}^{12}$ and from all reported cases, $841 \mathrm{ml}(n=51)$. Patients suffered preceding symptoms for a mean of 39 days prior to presentation (range 0-180 days). Right ventricular diastolic collapse on echocardiography or cardiac catheterization is evident prior to the development of hypotension or pulsus paradoxus, and mean arterial pressure is maintained in the early stages of tamponade.

Pericardial seeding by malignant cells is probably insufficient to cause tamponade: lymphatic obstruction seems to be an additional requirement. As described by Fraser et al., lung tumours spread to mediastinal lymph nodes, from where retrograde spread occurs to the epicardial lymphatic plexus. Slow accumulation of large volumes of fluid may then occur. Tumours which metastasize primarily by the haematogenous route, or those that rarely spread to mediastinal nodes rarely cause tamponade. Many tumours that metastasize to heart or pericardium very rarely present with tamponade through a combination of lack of lymphatic obstruction and earlier presentation with local symptoms, for example, breast, colon, pancreas, stomach, ovary, melanoma. Similarly, squamous carcinoma of the lung may be a rare cause of tamponade as it is typically endobronchial and symptoms of haemoptysis, cough or bronchial obstruction may cause earlier presentation than adenocarcinomas, which are more usually situated distally in the pulmonary parenchyma.

The most common clinical features of malignant cardiac tamponade are non-specific. Since malignant tamponade usually develops as an acute event superimposed on a background of a large, slowly developing pericardial effusion, the clinical appearance is often more suggestive of congestive cardiac failure. In a review of malignant tamponade pooling several series, ${ }^{1}$ the most common symptoms were dyspnoea (79\%), cough $(47 \%)$, thoracic pain $(27 \%)$, orthopnoea $(26 \%)$ and dysphagia $(18 \%)$. Physical signs encountered were pleural effusion $(51 \%)$, tachycardia $(50 \%)$ and signs of systemic venous congestion such as elevated jugular venous pressure, hepatomegaly and peripheral oedema. Signs considered typical of pericardial effusion - pulsus paradoxus, a pericardial rub and Kussmaul's sign - occurred in only $30 \%, 12 \%$ and $5 \%$, respectively. Although abnormal ECGs were reported in over $90 \%$, the changes were all non-specific save electrical alternans which occurred in only $5 \%$. The need to maintain a high index of suspicion in patients presenting with apparent cardiac failure and hypotension of unknown aetiology is vital if the diagnosis is not to be delayed. Delay in diagnosis of cardiac tamponade, even in the face of typical clinical features was a feature of our series. This has been noted in other reports. ${ }^{12}$ Earlier and more widespread use of echocardiography in the investigation of patients with apparent cardiac failure may facilitate diagnosis. The assumption of an ischaemic aetiology is not necessarily safe even in patients with known coronary artery disease, as illustrated by case 4 , and the administration of angiotensin converting enzyme inhibitors to patients with extrinsic myocardial compression may precipitate sudden circulatory collapse.

The immediate drainage of pericardial fluid is life saving and brings about symptomatic relief. It also provides a diagnosis of malignancy in the majority of cases. In reported cases, aspirated fluid was haemorrhagic in 59 of 67 cases where information was available $(88 \%)$ and cytology positive in 56 of $67(84 \%)$. Measurement of carcinoembryonic antigen (CEA) in pericardial fluid may provide additional diagnostic help, as elevated levels are found in many malignant effusions. In one series, a combination of cytology and CEA measurement was $100 \%$ sensitive and specific for malignancy. ${ }^{13}$

Subacute management of malignant pericardial effusion requires either sclerosis of the pericardial space or pericardial fenestration. Operative peri- 8 cardial fenestraton requires thoracotomy under general anaesthesia and unsurprisingly carries an $\bar{c}$ immediate mortality of approximately $8 \% .^{14} \mathrm{~A} \rightleftharpoons$ safer procedure with low recurrence rates (approximately $3 \%$ ) is subxiphoid pericardiotomy, which may be performed under local anaesthetic. ${ }^{1}$ A more recently developed alternative is balloon catheter pericardiotomy, first reported in 1991 by Palacios et $a .^{15}$ and critically reviewed by Keane and Jackson. ${ }^{16}$ This technique effected prolonged relief of tamponade in $94 \%$ of a series of 35 patients. ${ }^{17}$ Its place in the management of patients with malignant effusions is not clear at the present time as considerable operator skill is required, the equipment is costly, and results are not presently superior to subxiphoid pericardiotomy. Tetracycline instillation after pericardial drainage has been successful in two reported series, ${ }^{18,19}$ but a mean of three instillations over the course of several days is required and an in situ drain was needed for a mean of 9 days. The use of corynebacterium parvum as a sclerosant has not previously been reported for pericardial effusion but it is commonly used for pleurodesis.

Whilst the prognosis for patients presenting with cardiac tamponade is poor, survival for several months is the norm and prolonged survival may be possible. Including our patients, mean survival for all patients in whom survival times are quoted in the literature was 144 days $(n=66$, range $4-1,400$ days). Prolonged survival in those patients with 
tumours amenable to systemic chemotherapy, for example, haematological and gynaecological malignancies, is possible. Ten patients $(13 \%)$ in reported series survived for over a year. Chemotherapy alone did not prolong survival in one series, ${ }^{12}$ but combined radiotherapy and chemotherapy did.

We would suggest that the occurrence of cardiac tamponade as the first manifestation of malignancy is more common than hitherto appreciated. A high degree of suspicion is necessary, but all of our cases could have been identified by clinical features and careful review of radiographic and electrocardiographic data. Echocardiography should be mandatory in patients with apparent cardiac failure where aetiology is unclear. Pericardiocentesis will provide

\section{References}

1. Press, O.W. \& Livingston, R. Management of malignant pericardial effusion and tamponade. JAMA 1987, 257: 1088- 1092.

2. Thurber, D.L., Edwards, J.E. \& Achor, R.W.P. Secondary malignant tumours of the pericardium. Circulation 1962, 26: 228-241.

3. Fraser, R.S., Viloria, J.B. \& Wang, N.S. Cardiac tamponade as a presentation of extracardiac malignancy. Cancer 1980 , 45: $1697-1704$.

4. Aggarwal, P. \& Sharma, S.K. Cardiac tamponade as the initial presentation of malignancy. Int J Cardiol 1989, 22: $157-159$.

5. Hwang, M.H., Brown, A., Piao, Z.E. \& Scanlon, P.J. Cardiac lymphoma associated with superior vena caval syndrome and cardiac tamponade: case history. Angiology 1990, 41: $328-332$.

6. Cercato, M.C., La Ferla, G., Pericoli, N., Fedele Scinto, A., Saccji, I. \& Cognetti, F. Cardiac tamponade as the initial manifestation of an extracardiac malignancy. Eur $J$ Cancer 1991, 27: 670-671.

7. Krisanda, T.J. Atrial fibrillation with cardiac tamponade as the initial manifestation of malignant pericarditis. Am J Emerg Med 1990, 8: 531-533.

8. Blanco, C., Cobo, J., Gomez-Cerezo, J., Ramos, F. \& Molina, F. Infarto agudo de miocardio y taponamiento cardiaco como manifestacion inicial de un adenocarcinoma. Rev Clin Esp 1990, 187: 210-211.

9. Rinkevich, D., Borovik, R., Bendett, M. \& Markiewicz, W. Malignant pericardial tamponade. Med Ped Oncol 1990, 18: 287-291.

10. Forslund, T., Forsen, K.O. \& Maenpaa, J. Cardiac tamponade due to ovarian carcinoma. Arch Gynecol Obstet 1991, 248: $161-165$. a diagnosis in over $80 \%$ of cases, and may allow diagnosis in an even greater proportion if cytology is combined with carcinoembryonic antigen measurement. Definitive treatment of the malignant effusion by means of subxiphoid pericardiotomy or percutaneous balloon pericardiotomy, or slerosant therapy with tetracycline may allow prolonged symptom-free survival. Combined chemotherapy and radiotherapy may prolong survival regardless of tumour type.

\section{Acknowledgement}

We wish to thank Dr A.J.S. Gardiner for his permission to report case 5 .
11. Sharma, S., Balaney, B. \& Mandke, J.V. Cardiac tamponade as a presentation of mucoepidermoid bronchogenic carcinoma. J Assoc Phys India 1989, 37: 183-185.

12. El Allaf, D., Burette, R., Pierard, L. \& Limet, R. Cardiac tamponade as the first manifestation of cardiothoracic malignancy: a study of ten cases. Eur Heart $J$ 1986, 7: 247-253.

13. Tatsuta, M., Yamamoto, R., Ichii, M., Lishi, H. \& Noguchi, S. Carcinoembryonic antigens in the pericardial fluid of patients with malignant pericarditis. Oncology 1984, 41: $328-340$.

14. Gregory, J.R., McMurtrey, M.J. \& Mountain, C.F. A surgical approach to the treatment of pericardial effusion in cancer patients. Am J Clin Oncol 1985, 8: 319-323.

15. Palacios, I.F. Percutaneous balloon pericardial window for patients with malignant pericardial effusion and tamponade. Cathet Cardiovasc Diagn 1991, 22: 244-249.

16. Keane, D. \& Jackson, G. Managing recurrent malignant pericardial effusions. Br Med J 1992, 305: 729-730.

17. Ziskind, A.A., Pearce, C., Burstein, S. et al. Percutaneous balloon pericardiotomy for the treatment of pericardial effusion and tamponade: report of the registry. $\mathrm{J} \mathrm{Am} \mathrm{Coll}$ Cardiol 1992, 19: 265A

18. Davis, S., Rambotti, P. \& Grignani, F. Intrapericardial tetracycline sclerosis in the treatment of malignant pericardial effusion: an analysis of 33 cases. $J$ Clin Oncol 1984, 2: 631-636.

19. Shepherd, F.A., Morgan, C.D., Ginsberg J.S. et al. Control of malignant pericardial effusion by tetracycline sclerosis. Proc Am Soc Clin Oncol 1986, 5: 246. 